Article

\title{
Experimental Study of Impingement Effusion Cooled Double-Wall Combustor Liners: Aerodynamic Analysis with Stereo-PIV
}

\author{
Thomas Jackowski ${ }^{\dagger, \ddagger}$, Maximilian Elfner ${ }^{*} \ddagger \oplus \mathbb{D}$, Hans-Jörg Bauer ${ }^{\ddagger}$, Katharina Stichling ${ }^{\S}$ and Marco Hahn $\S$
}

check for

updates

Citation: Jackowski, T.; Elfner, M.; Bauer, H.-J.; Stichling, K.; Hahn, M. Experimental Study of Impingement Effusion Cooled Double-Wall Combustor Liners: Aerodynamic Analysis with Stereo-PIV. Energies 2021, 14, 6191. https://doi.org/ 10.3390/en14196191

Academic Editors: Antonio Andreini and Lorenzo Mazzei

Received: 2 August 2021

Accepted: 23 September 2021

Published: 28 September 2021

Publisher's Note: MDPI stays neutral with regard to jurisdictional claims in published maps and institutional affiliations.

Copyright: (c) 2021 by the authors. Licensee MDPI, Basel, Switzerland. This article is an open access article distributed under the terms and conditions of the Creative Commons Attribution (CC BY) license (https:// creativecommons.org/licenses/by/ $4.0 /)$.
Institute of Thermal Turbomachinery (ITS), Karlsruhe Institute of Technology (KIT), 76137 Karlsruhe, Germany; thomas.jackowski@kit.edu (T.J.); hans-joerg.bauer@kit.edu (H.-J.B.); katharina.stichling@kit.edu (K.S.); marco.hahn@kit.edu (M.H.)

* Correspondence: maximilian.elfner@kit.edu; Tel.: +49-721-608-43634

+ Retired from Karlsruhe Institute of Technology (KIT).

$\ddagger$ These authors contributed equally to this work.

$\S$ These authors supported the review and editing of the article.

Abstract: A new experimental study is presented for a combustor with a double-wall cooling design. The inner wall at the hot gas side features effusion cooling with 7-7-7 laidback fan-shaped holes, and the outer wall at the cold side features an impingement hole pattern with circular holes. Data are acquired to asses the thermal and aerodynamic behavior of the setup, using a new, scaled up, engine similar test rig. Similarity includes Reynolds, Nusselt and Biot numbers for hot gas and coolant flow. Different geometrical setups are studied by varying the cavity height between the two walls and the relative alignment of the two hole patterns at two different impingement Reynolds numbers. This article focuses on the aerodynamic performance of the setup. Instationary flow data are acquired, using a high speed stereo PIV setup. For each geometrical configuration, approximately 20 planes are recorded with a data rate of $1000 \mathrm{~Hz}$ by traversing the flow region of interest in the cavity between the two specimen. This fine resolution allows the reconstruction of 3D flow fields for the mean data values and an extensive analysis of transient phenomena at each plane. Time averaged data and jet-center plane transient data are presented in detail. The results show a complex flow field with a hexagonal vortex pattern in the cavity, which is mainly influenced by the cavity height and the relative alignment of the two walls. The jet Reynolds number shows small influence when analyzing normalized data. Small cavity heights show a less developed flow field with less stable vortex systems. The alignment shows a similar influence on vortex system stability, with the aligned case performing better. Additionally, statistical analysis of the jet flow and frequency domain analysis of the jet and the effusion flow are presented, showing the damping capability of the cavity, especially at increased cavity heights, and a residual low frequency pulsation of the effusion cooling inflow.

Keywords: combustor cooling; effusion impingement; PIV

\section{Introduction}

The continuous development of aero-gas turbines leads to constant improvements that benefit air passengers and the environment. A commonly used combustion technology to reduce the formation of $\mathrm{NO}_{\mathrm{x}}$ is the RQL combustor, describing the three major combustion regions-Rich, Quench and Lean-in an annular combustor. As shown in Figure 1, the air from the compressor is divided into two major flow paths. A small amount of the compressor air is used to create an air-assisted fuel spray, providing a minimal amount air for burning that fuel spray in a rich environment (red arrow). The remaining air is guided around the combustor liner and used for consecutive mixing, dilution and, mainly, for the cooling of the liner walls. 


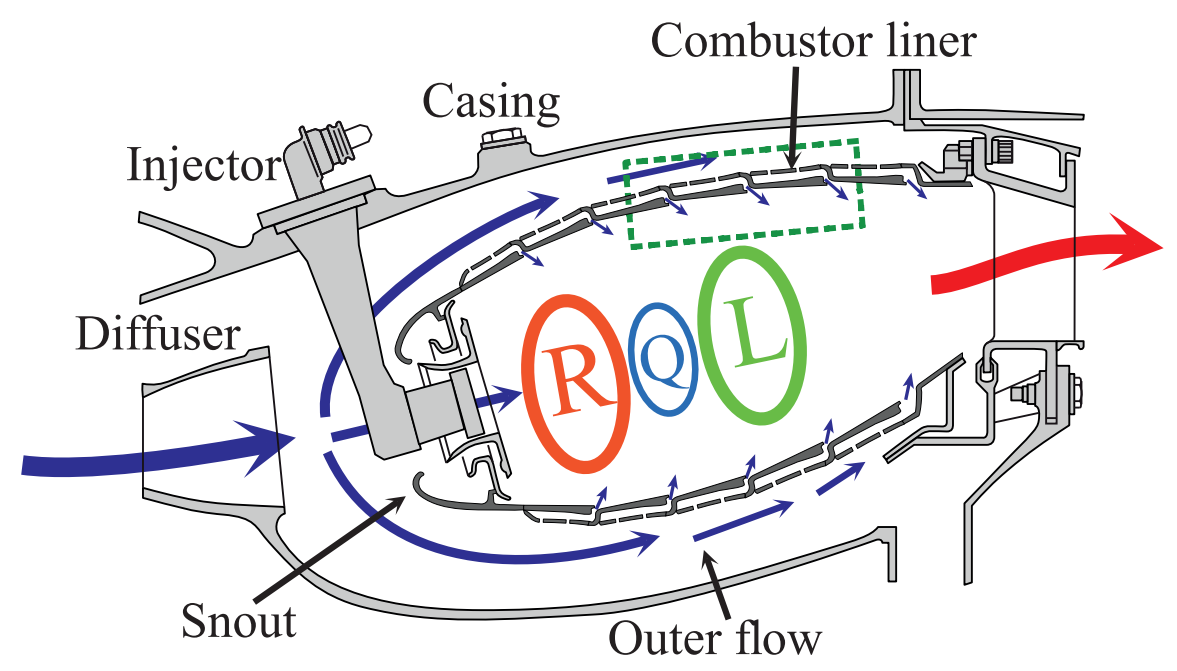

Figure 1. Double-wall design study adapted from V2500 combustor. The compressed air (blue) is divided between the combustor liner and outer flow. The air in the outer flow acts as mixing air and coolant, while the combustor liner air is used for primary combustion (red). Green area marks the region of interest for this study with focus on the cavity between the two liner walls.

Many different cooling schemes exist to allow for protection of the combustor walls, which are exposed to flame temperatures varying from 1700-2500 K. A good overview is given in [1] (p. 3). With a pressure drop of $\Delta p / p \approx 3-4 \%$ between the liner and outer flow, impingement-effusion cooling can protect the metal parts efficiently with higher cooling effectiveness. This concept implements a double wall for the combustor liner, where an initial stage of impingement cooling is followed by an effusion cooling scheme for the innermost wall. The flow in between the two specimen is complex and has great influence on especially the performance of the effusion cooling.

This article presents an extensive experimental investigation of the aerodynamic behavior of such a cooling design. An engine-similar test rig is presented, which allows the analysis of flow in the cavity between the two liner walls, using particle image velocimetry (PIV) at engine similar conditions. The presented results show the effects of varying operating conditions and different geometrical setups, mainly the cavity height between the double walls and the relative positioning of impingement and effusion holes at different impingement Reynolds numbers. Time averaged and transient results are presented, adding to the understanding of the complex cavity flow and its effect on thermal performance.

A second article with an extensive thermal analysis using thermography has been prepared and published [2].

\section{Flow Analysis in Impingement Effusion Cooling Double-Walls}

Andrews et al. [3] carried out experiments to investigate the overall wall heat transfer for an impingement and effusion cooled wall. The authors concluded an increased heat transfer with both cooling methods combined, compared to applying impingement cooling alone; however, effusion cooling has a larger contribution. Different impingement and effusion double wall configurations also were experimentally investigated by Martiny et al. [4] under scaled conditions and realistic density ratios. The results also show a major influence of effusion cooling especially downstream on the test surface.

El-Jummah et al. [5] investigated the same topic by performing conjugate heat transfer calculations with CFD methods. A similar calculation was done by Oguntade et al. [6] with reduced mass flows. All authors agree that impingement cooling can increase total cooling effectiveness, especially in the early upstream effusion region where the exterior coolant film is not yet fully deployed. The main cooling contribution, especially downstream, is introduced by the effusion cooling on the hot gas side. Thus, significant parts of the flow 
design for a double wall cooling need to ensure a homogeneous and effective feed of the effusion holes while maintaining an acceptable impingement cooling effectiveness.

Different plate distances between impingement and effusion wall were studied by Al Dabagh et al. [7]. Additionally, a variation of hole size by maintaining the amount of holes was performed. They showed strong influence of the wall distance on the resulting flow field and effusion cooling infeed. Cocchi et al. [8] investigated two geometries of a double wall cooling scheme experimentally and using CFD. Hole pattern geometries were comparable with the ones used in this study. They showed an influence of longitudinal pattern shift on cavity flow patterns. Flow analysis showed hexagonal flow patterns comparable to cloud structures, influencing the effusion hole infeed and subsequently, the discharge coefficient. Chen et al. [9] carried out CFD calculations for a similar cooling setup, but introduced crossflow for the coolant between the inner and outer walls. The results are comparable.

In [10-12], a series of experiments were carried out to investigate full-coverage effusion cooling of a combustor wall with internal impingement cooling. The results are in agreement with previous studies with the main new feature being the simultaneously acquired data on either side of the effusion specimen. Crossflow leads, in general, to a deterioration of hot side cooling effectiveness. Flow analysis is only performed with basic probes and derived from thermal data but again shows a complex flow field in the cavity between the two walls.

Murray et al. $[13,14]$ conducted numerical and experimental investigations for walls with combined impingement and effusion cooling methods designed for turbine blades and developed methods to evaluate the cooling performance of cooling schemes with double walls. Using CFD, they showed complex flow fields in the cavity between the two walls and also showed the interaction between impingement flow and effusion hole flow.

Shrager et al. $[15,16]$ performed thermal and aerodynamic analysis of a double-wall combustor liner. With a general impingement-effusion setup comparable to the one in this study, they focused on the area of dilution holes which are not included in this article's setup. The time-averaged results are shown for the host gas side effusion flow.

Jet impingement heat transfer with a focus on the correlation to flow analysis was performed in $[17,18]$. While the first article shows steady-state results in good agreement to the data presented here, the second article shows transient data, especially at higher frequencies acquired using laser Doppler anemometry. Numerical data with obstructions (ribs) at the impingement surface are shown in [19]—however, only time-averaged values are shown.

The test setup used for this article was analyzed, using a conjugated heat transfer numerical approach [20]. The results indicate that the strongest effect on cooling effectiveness is introduced by the blowing ratio. After a short distance downstream of the initial effusion holes, the effusion cooling is of higher importance than the internal impingement cooling. The flow inside the cavity has a strong influence on the effusion cooling flow. It subsequently mainly depends on the cavity height and the arrangement between impingement holes (and their main flow trajectory) and effusion inflow holes.

A tabulated overview of available literature with research on double-wall combustor cooling is given in the appendix; see Appendix B.

\section{Experimental Methodology}

The test rig consists of two rectangular channel sections that are connected together through a cavity in which a combustor double wall is simulated. Measurements focus on the flow patterns in this cavity. The double wall cooling test section is integrated in the flow lab at the ITS. The infrastructure to drive the test section is shown in Figure 2, left side. For the open loop main flow the air is provided by a radial compressor, which is capable of providing a mass flow of $3 \mathrm{~kg} / \mathrm{s}$ at a pressure ratio of 1.4. The air can be heated by electrical heaters with a maximum power of $450 \mathrm{~kW}$, which is not used for the aerodynamic tests. After the heater, the air passes additional mixers, screens and meshes to further 
improve uniformity. Eventually, the airflow is accelerated through a nozzle to achieve the target velocity.

The coolant air is provided in a semi-closed loop. Compressed air is supplied by a rotary screw compressor and discharged through a valve into the closed loop coolant system. The amount of air introduced into the closed loop is tracked by a mass flow meter and equals the ejected coolant in the test section.

To circulate the air in the closed-loop section and to achieve engine similar coolant Reynolds numbers, a blower is integrated. To maintain constant air temperature, the heat input of the blower is removed with a heat exchanger.

(a)

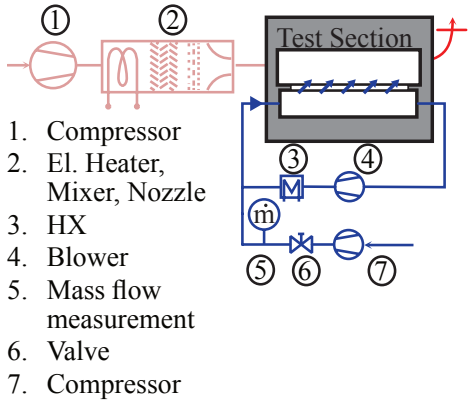

(b)

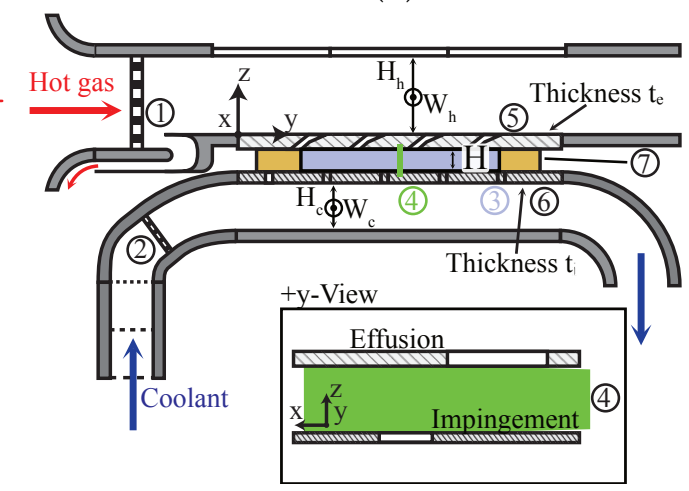

Figure 2. Test facility at ITS showing the hot gas path in red and the coolant path in blue. (a) Infrastructure (with object labels), (b) test section with (1) turbulence grid (2) meshes, screens and turbulence grid (3) glass frame inset (4) light sheet (5) specimen with effusion cooling pattern (6) specimen with impingement cooling pattern (7) PEEK frame around cavity.

\subsection{Test Section}

The central test section is an upscaled, planar double-wall combustor liner design, shown in Figure 2, right side. Both the hot gas and the coolant channels are rectangular in the cross section with parallel flow direction. They are connected via a cavity with an impingement plate on the coolant side and an effusion plate on the hot gas side.

The hot gas is introduced via the nozzle at the heater outlet. The exit width and height of the nozzle are $W_{\mathrm{h}}=500 \mathrm{~mm}$ and $H_{\mathrm{h}}=250 \mathrm{~mm}$. A turbulence grid generates an isotropic turbulence level of $T u=9.8 \%$ (design [21]). Further downstream, a boundary layer bleed is installed to obtain a reproducible boundary layer thickness in the hot gas channel. After the boundary layer bleed, the channel height reduces to $H_{\mathrm{h}}=231 \mathrm{~mm}$. The test specimen is integrated in the bottom wall of the channel.

The coolant channel has the same width and a height of $H_{c}=150 \mathrm{~mm}$. The coolant flow channel inlet design (see (2)) is adapted to increase coolant flow homogeneity (numerically designed and experimentally validated). The flow profile is further improved by introducing screens and meshes into the vertical portion of the coolant inlet. A turbulence grid with $\mathrm{Tu}=5.0 \%$ is added in the flow channel.

The connecting cavity is introduced through a plastic frame and sealed with PTFE against both channels. The height of the inset defines the distance between the specimen as specified for the different geometrical setups. A shift in longitudinal direction between impingement and effusion specimen can be set. The geometrical configurations shown in this article are summarized in Table 1. To allow for optical measurements in the cavity using PIV, parts of the PEEK frame can be replaced by glass elements. Seeding is introduced to the coolant flow far upstream of the initial mixers and turbulence grid to allow for a homogeneous particle distribution. 
Table 1. Overview of geometrical test configurations.

\begin{tabular}{lcc}
\hline & Normal Al. (MALO00) & Shifted Al. (MALO05) \\
\hline Cavity Height $H$ & 3D, 5D, 7D & 3D, 5D, 7D \\
Longitudinal Shift $+S_{y}$ & 0.0 & $0.5 S$ \\
Coolant Imp. Re $\mathrm{D}_{\mathrm{D}, \mathrm{imp}}$ & $10,620,17,170$ & $10,620,17,170$ \\
\hline
\end{tabular}

In contrast to the thermal experiments, PIV experiments are performed with nonheated main flow. This reduces cavity flow temperatures and high-speed camera temperatures, both leading to a major increase in PIV raw image quality. However, the density ratio is lower at $\mathrm{DR} \approx 1$, which can be accepted since the main area of interest is the flow in the cavity. Thus, the experiments are not performed at a multitude of blowing ratios (or similar momentum flux ratios I) but at different coolant impingement Reynolds numbers-this Reynolds number is identified as the main similarity for the cavity flow structures, while the momentum flux ratio mainly influences interaction with the hot gas flow. The coolant crossflow and hot gas Reynolds numbers are still chosen to match those of the thermal experiments.

Comparing the operating points with the thermal measurement operating points should thus be performed considering Reynolds numbers since blowing ratios and momentum flux ratios vary, due to changes in density and velocity of the hot gas flow. The Reynolds numbers are computed with the following:

$$
\operatorname{Re}_{\mathrm{D}, \mathrm{imp}}=\frac{4 \dot{m}_{\mathrm{tot}}}{\pi D N v}
$$

with the total impingement mass flow and the total area of all impingement holes combined ( $N$ number of holes, 159). The viscosity is determined using a Sutherland approach with the coolant inlet temperature $T_{c}$. The Reynolds number is controlled employing the mass flow rate. The operating points chosen are a high nominal impingement Reynolds number of $\operatorname{Re}_{\mathrm{D}, \mathrm{imp}}=17,170$ and a low impingement Reynolds number of $\mathrm{Re}_{\mathrm{D} \text {, imp }}=$ 10,620 . While the former corresponds to the high blowing ratio of $M \approx 3$ in the thermal experiment, the latter corresponds to the same blowing ratio at unscaled engine conditions, including non-linear effects in the material properties introduced with the large change in temperatures during scaling. The operating conditions are summarized in Table 2.

Table 2. Overview of operating conditions. Data are shown for both nominal impingement Reynolds numbers. Deviation of values over all six different test configurations given for each Reynolds number.

\begin{tabular}{|c|c|c|}
\hline Flow Conditions & $\begin{array}{l}\text { Coolant Imp. Re } \\
\operatorname{Re}_{\mathrm{D}, \mathrm{imp}}=10,620\end{array}$ & $\begin{array}{l}\text { Coolant Imp. Re } \\
\operatorname{Re}_{\mathrm{D}, \text { imp }}=17,170\end{array}$ \\
\hline Hot gas Inlet $T_{\mathrm{h} \text {, tot }}$ & $335 \mathrm{~K} \pm 1.5 \%$ & $334 \mathrm{~K} \pm 1.5 \%$ \\
\hline Hot gas $\operatorname{Re}_{\mathrm{D}, \mathrm{h}}$ & $1.962 \pm 0.6 \%$ & $1.948 \pm 0.6 \%$ \\
\hline Hot gas $\mathrm{Tu}_{\mathrm{h}}$ & $9.8 \%$ & $\leftarrow$ \\
\hline Coolant Inlet $T_{\mathrm{C}}$ & $296 \mathrm{~K} \pm 0.2 \%$ & $295 \mathrm{~K} \pm 0.3 \%$ \\
\hline Coolant Imp. $\operatorname{Re}_{\mathrm{D}, \mathrm{imp}}$ & $10.458 \pm 0.3 \%$ & $17.024 \pm 0.5 \%$ \\
\hline Comp. Jet Exit Velocity $v_{\text {jet,e }}$ & $39.6 \mathrm{~m} / \mathrm{s} \pm 0.9 \%$ & $62.3 \mathrm{~m} / \mathrm{s} \pm 0.8 \%$ \\
\hline Coolant Crossflow $\operatorname{Re}_{\mathrm{D}, \mathrm{c}}$ & $4.247 \pm 0.3 \%$ & $4.246 \pm 0.4 \%$ \\
\hline Coolant $\mathrm{Tu}_{\mathrm{c}}$ & $5 \%$ & $\leftarrow$ \\
\hline \multicolumn{3}{|c|}{ Derived values at cold test rig conditions } \\
\hline Density Ratio $D R$ & $1.15 \pm 1.3 \%$ & $1.18 \pm 1.3 \%$ \\
\hline Eff. Blowing Ratio $M$ & $2.46 \pm 1.1 \%$ & $4.0 \pm 1.0 \%$ \\
\hline Eff. Momentum Flux Ratio I & $5.24 \pm 3.2 \%$ & $13.6 \pm 3.0 \%$ \\
\hline
\end{tabular}


Compared to the thermal operating points, slightly higher deviations are visible. Those are mainly introduced by a varying hot gas temperature, which is not perfectly controlled when the main heater is not used. However, considering that the measurements were performed over multiple days, the operating points are highly stable and thus, the data are well comparable.

\subsection{Test Specimen}

The used scaling factor for a typical engine geometry is $S F=8$, resulting in a reference diameter of $D=4 \mathrm{~mm}$. The specimen on the hot gas side with effusion holes has 159 laidback fan-shaped cooling holes distributed over 25 rows. In streamwise direction, the effusion hole pattern is shifted by $P / 3$, i.e., the pattern repeats every 4 th row. In streamwise direction, every row of cooling holes has a distance of $S=6.67 \mathrm{D}$ to the next row. In a row, the pitch between each cooling hole is $P=10 D$. The effusion cooling holes are inclined by $\alpha=30^{\circ}$ to the surface and have a cylindrical entry diameter of $E=\sqrt{2} D$. The lateral opening angle and laidback opening angle is $\beta=7^{\circ}$ (designed after the 7-7-7 configuration introduced by Schroeder et al. [22]).

The impingement specimen has vertical cylindrical cooling holes with a diameter of $D$. The pitch and spacing of each cooling hole remain the same as for the effusion cooling holes, while their position is offset as described in Figure 3a. The chosen placement maximizes the wetted area on the backside of the effusion specimen before the coolant is ejected through the effusion cooling holes. For the misaligned setup $M A L O 05$, the impingement specimen is shifted by $S_{y}=0.5 P$ in longitudinal direction $(y)$, placing the impingement exit opening on the rows of effusion entry holes; see Figure $3 \mathrm{~b}$. The specimen with the effusion holes is made of TiAl6-V4 and has a thickness of $t_{\mathrm{e}}=12 \mathrm{~mm}$. The impingement plate is made of stainless steel AISI 420 with a thickness of $t_{\mathrm{i}}=8 \mathrm{~mm}$. The selection of material and thickness were derived from scaling the Biot number for a flat plate at jet engine conditions to experimental conditions. Additional information on hydraulic porosity and similarity scaling for the two specimen are given in the article focusing on the thermal study [2].

(a)

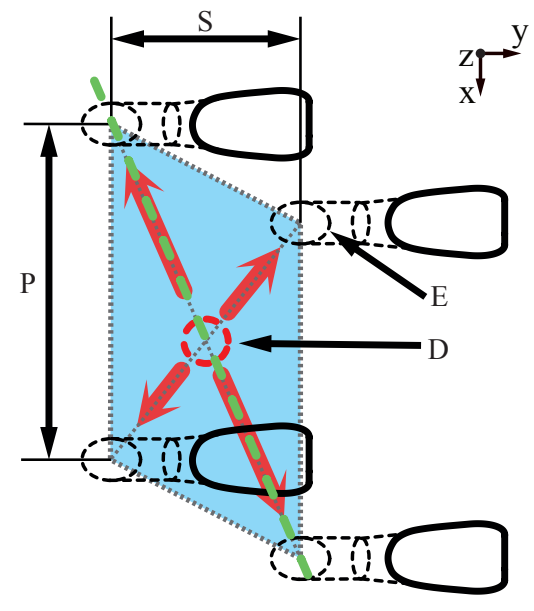

(b)

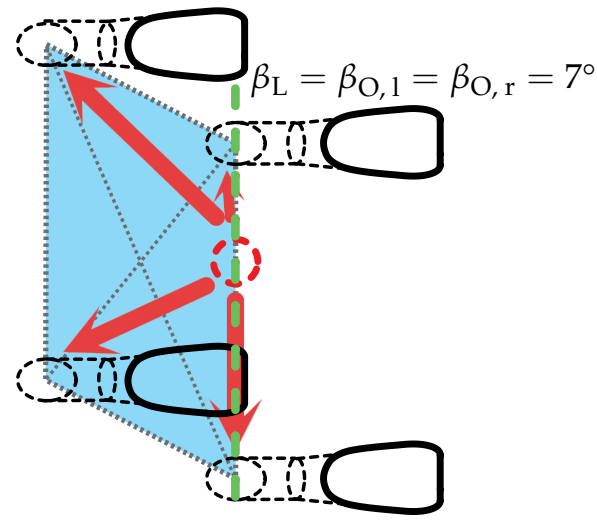

Figure 3. (a) Arrangement of each impingement cooling hole to the effusion cooling holes. Red arrows show theoretical exit paths and distance to the next effusion cooling hole. The blue parallelogram shows the wetted area before the coolant is ejected. (b) Misaligned setup MALO05. Cut planes for later evaluation shown in green.

\subsection{Measurement Methods and Data Processing}

The velocity data are acquired, using high speed stereo PIV. The setup consists of a Quatronix Darwin Duo $527 \mathrm{~nm}$ green dual cavity laser with a power of $P_{\text {Laser }} \approx 100 \mathrm{~W}$ and two Photron Fastcam SA4 cameras. The synchronization is set to a data rate of $f_{\mathrm{d}}=1000 \mathrm{~Hz}$ 
, allowing frequency analysis up to $f_{\mathrm{f}, \max }=500 \mathrm{~Hz}$. Considering RAM limitations and frame size (depending on cavity height), the total number of images and thus, the total amount of recorded time, varies from 4365 images per plane for the large cavity height of $7 D$ up to 5456 images per plane for the small height $3 D$. Average values do not change significantly after an initial set of $\approx 1200$ images for any of the configurations.

The laser sheet optical axis is aligned with the $x$-Axis. The cameras are placed approximately at the laser outlet $(x)$ and offset to the $\pm y$-axis. The stereo angle is $\approx 35^{\circ}$ in a sideways-backward scattering setup. The cameras and the laser are placed on a traverse system, which moves the combined setup in $y$-direction. For each test configuration, approximately 20 light sheets are recorded with a spacing of $\Delta y_{1 \mathrm{~s}} \approx 2 \mathrm{~mm}$ for each configuration $(\approx 250$ total). The resulting sheet setup (center sheet) is shown in Figure $4 \mathrm{a}$. Due to the high spatial resolution, time averaged data for each test configuration can be re-interpolated in $3 \mathrm{D}$ space, using a linear interpolation on a regular grid with a spacing of $\approx 0.3 \mathrm{~mm}$.

(a)

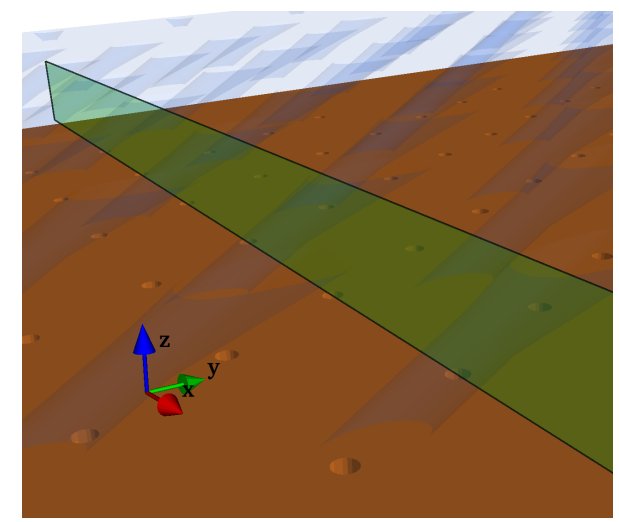

(b)

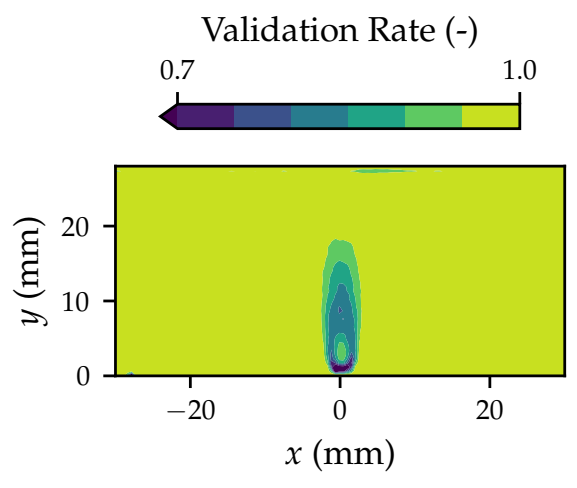

Figure 4. (a) Light sheet arrangement in the cavity. Impingement specimen in brown, effusion specimen in blue. Center sheet location relative to impingement hole for $M A L O 00$ case. (b) Validation rate for a worst case scenario $7 D-M A L O 00$, high $\operatorname{Re}_{\mathrm{D} \text {, imp, }}$, center plane.

The origin is placed at the outlet of the impingement hole for the MALO00 cases. For MALO05 cases, the relative alignment to the impingement holes changes, e.g., the sheets are kept at the same absolute positions. The hole outlet thus is subsequently located at $\{x, y, z\}=\{0, S / 2,0\}$.

Calibration is performed a single time for each test configuration before acquiring all data planes, using a multi layer target placed inside the test rig and the PIVView software package. Subsequently, the images are post-processed using the same software. Post processing includes a disparity correction and a standard multipass PIV evaluation with a final grid size of $16 \mathrm{px}$ and a window overlap of $50 \%$, yielding a final physical resolution of 2 vecs. $/ \mathrm{mm}$. Outliers (points with no stable correlation result) are identified but not replaced, using, e.g., interpolation at this stage of evaluation.

The time distance for the double images is chosen for each case to optimize validation rate between $5 \mu \mathrm{s}-10 \mu \mathrm{s}$. The resulting validation rates are shown for a worst case scenario with high Reynolds number at large cavity heights in the center plane (this yields the maximum span of velocities in the domain) in Figure $4 \mathrm{~b}$. Validation rates are in unity in most of the domain; however, in the core jet, some evaluations fail, due to high velocity and thus, high particle displacement. Before final processing, these remaining outliers are interpolated, using a spatial linear approach at each time step. Afterwards, only points with a validation rate of unity are considered-this removes points at borders where the convex hull of valid data changes with time and points inside the domain if interpolation errors occur, yielding the most conservative approach. The data can then be used for transient analysis or 3D interpolation and visualization. 
For estimating the measurement uncertainty in the PIV experiments, a method based on correlation statistics introduced by [23] was used. The local relative uncertainty was found to be below $5 \%$ in the core jet region. The spatial average of the temporally averaged relative local uncertainty in the impingement jet main stream direction was below $16 \%$. This high value is mainly caused by locally very small displacements. Due to the complexity of the three-dimensional impingement flow field this cannot be avoided.

This uncertainty analysis is based on the time-resolved data. As stated above, after employing approximately $25 \%$ of available time resolved data, the mean values and statistics of the data do not change significantly. Thus, the error (excluding a possible bias) is further reduced in these data set types.

\section{Results and Discussion}

The presented experimental setup allows the acquisition of time-resolved velocity data in the cavity of a double-wall combustor liner. Compared to previous work, several unique features are realized in this experimental setup. Coolant cross flow is introduced, mainly affecting the impingement jets. Two main geometrical variations are identified and studied: the change in cavity height as a main design parameter and the relative alignment of effusion to impingement specimen, which can occur due to thermal expansion in the real engine.

The results are discussed in the following sections: initially, time-averaged velocity data are analyzed to show jet profiles for different configurations, the effects of cavity height and longitudinal alignment on the flow patterns inside the cavity. Core influence factors are identified. Subsequently, several configurations are chosen and compared against the baseline configuration of $H=5 D, M A L O 00$ (which also showed good thermal performance). The comparison is performed, applying more advanced techniques and leveraging the time-resolved data.

Most of the data, especially velocities, are shown in normalized quantities:

$$
v_{\mathrm{N}}=\frac{v}{v_{\text {ref }}}
$$

normalized with, for example, the reference velocity $v_{\text {ref }}$ being the maximum value of the dataset underlying the displayed case (e.g., maximum value of a given plane) if not otherwise stated. The maximum values for each plot are summarized in Appendix C.

\subsection{Jet Profiles}

Jet profiles are analyzed at the jet center, and thus, in different absolute locations for the aligned and misaligned cases. The figures show normalized jet velocity over lateral expansion. Profiles are shown at $20 \%, 50 \%$, and $80 \%$ relative cavity height $H^{*}$, which is defined as the following:

$$
H^{*}=\frac{H}{H_{\mathrm{des}}}
$$

with the three design cavity heights $H_{\text {des }}$ of $3 D, 5 D$ and $7 D$. The aligned case $M A L O 00$ is shown with solid lines, the misaligned case with dashed lines. All profiles are displayed in Figure 5. Additionally, the profiles at the same origin but on $x$-normal planes are shown in Figure A1 in Appendix A (slightly lower resolution due to out of plane direction on plot $x$-axis). 


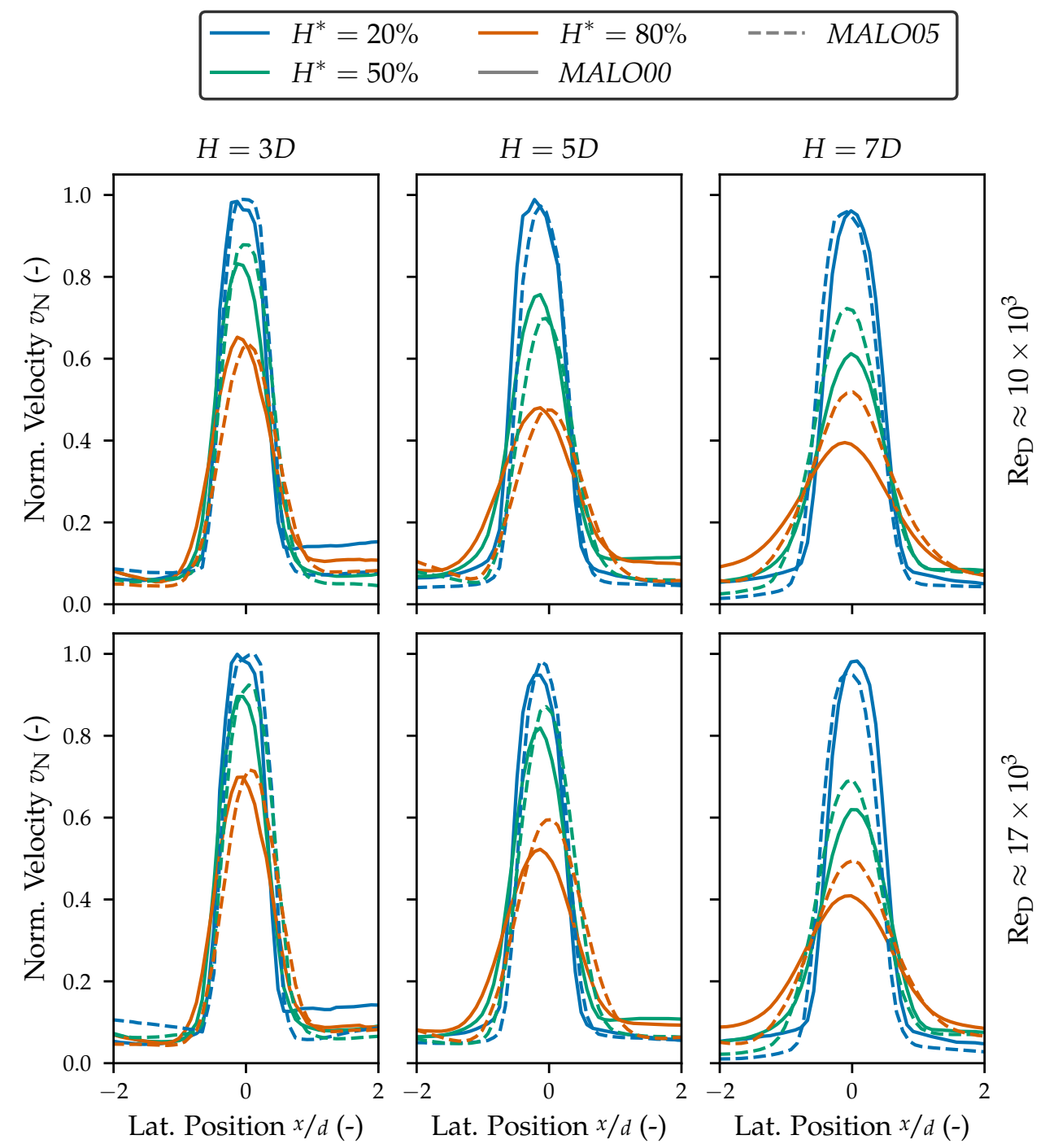

Figure 5. Jet profiles for all configurations tested. Profiles shown normalized velocity at three different height (colors) for both alignments (line style) at both Reynolds numbers (rows) and different cavity heights (columns). Velocities taken at $y$-normal planes.

All configurations show a distinct jet profile with the highest velocities at the exit location and a degradation of the jet downstream, e.g., the maximum velocity drops while the jet width increases. The profiles are similar for all configurations, and thus, no major changes in flow regime are induced by the changing test configurations. At the smallest cavity height $H=3 D$, the jet profile shows little momentum loss, with peak velocities at $H^{*}=0.8$ of above $v_{\mathrm{N}} \geq 0.6$, leading to a high local heat transfer at the effusion target plate. With increasing cavity height of $H=5 D$ to $H=7 D$, the jet momentum loss increases gradually, leading to wider jet profiles with lower peak velocities. This results in a more equalized jet heat transfer at the effusion target wall. The presence of misalignment (cases MALO05) shows a major effect with larger cavity heights at jet distances further downstream. The lower momentum jet is more strongly influenced by the changing flow patterns close to the effusion specimen, preventing momentum loss and inducing minor lateral shifts. The effects of alignment are, however, comparatively small.

The jets are symmetric in the $y$-normal plane. As shown in Figure A1, a jet deflection in the $x$-normal plane (thus in $y$-direction) is visible. The effect, again, increases with larger jet traveling distances and lower jet momentum (lower impingement Reynolds number). The jets are deflected in the negative main flow direction, induced by a separation bubble at the impingement hole inlet, due to the present crossflow, also shown in the preliminary 
CFD study [20]. While the effect is small with deflections below $0.5 D$, the position of the maximum heat transfer on the effusion specimen will vary. The effect of misalignment is very small. Only for large cavities and low momentum jets, most noticeably at $H=7 D$, $R e_{\mathrm{D}} \approx 10 \times 10^{3}$, the misalignment leads to jet stabilization as seen in the rotated plane in Figure 5 .

\subsection{Cavity Flow Patterns}

Depending on the configuration, distinct flow patterns in the cavity are expected, which can show a strong influence on the local heat transfer and effusion hole flow patterns. Due to the highly three-dimensional nature of the flow, some conclusions cannot be fully supported with the displayed figures. As stated below, the full datasets are available on request. The main jet velocity component $(z$-axis, $w)$ is shown at a constant height of $H^{*}=90 \%$ for all cavity heights and both alignments in Figure 6.
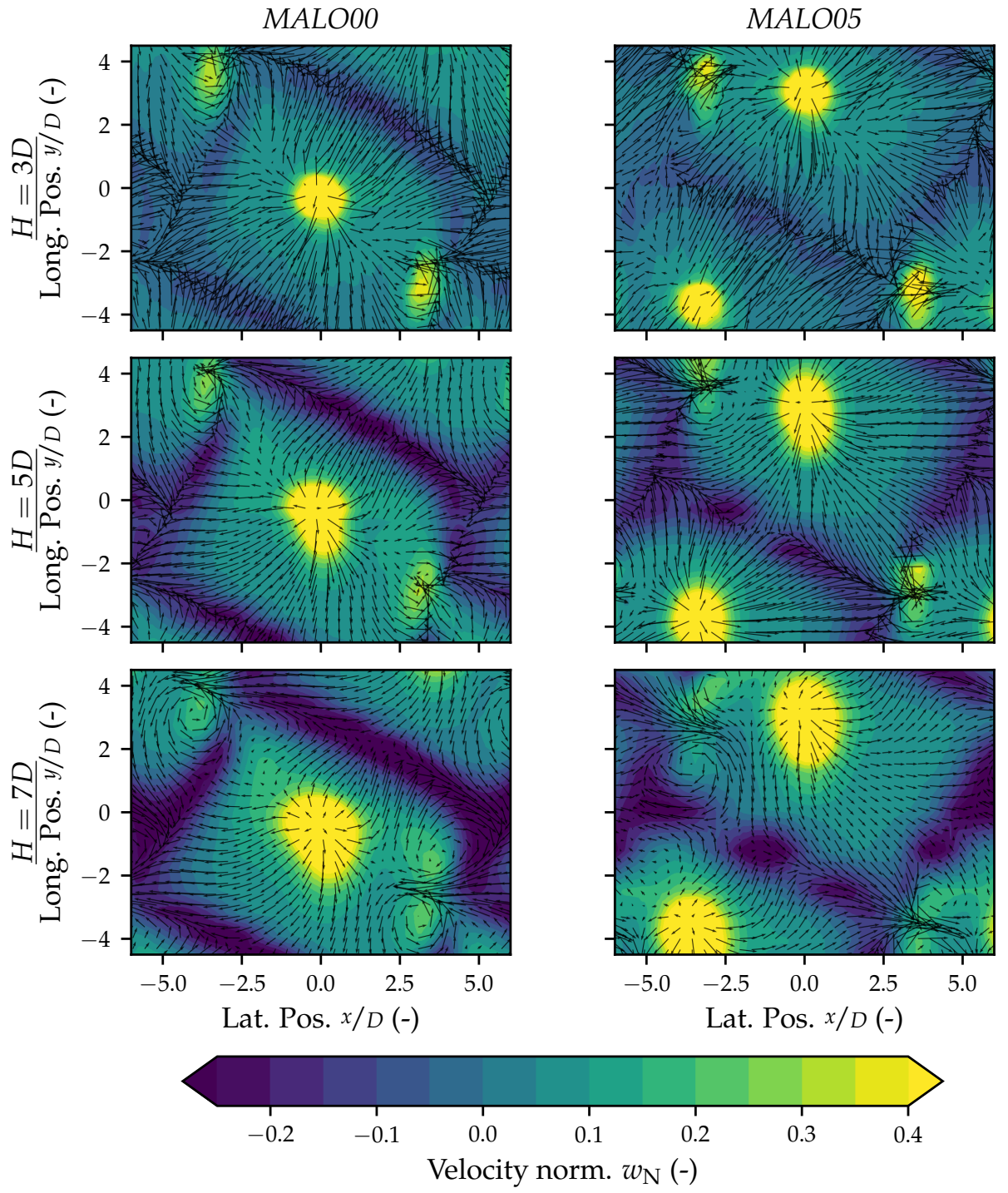

Figure 6. Cavity flow, low Reynolds number $R e_{\mathrm{D}} \approx 10 \times 10^{3}$. w-velocity normalized shown at $z$-normal plane with $H^{*}=80 \%$. Vectors show planar velocity components, unit length $v e c_{\mathrm{ul}}=3$. Rows show cavity height, columns show alignment.

The contour plots show normalized $w$-velocity (normalized with the max. velocity magnitude in a given plane) for the lower Reynolds number $R e_{\mathrm{D}} \approx 10 \times 10^{3}$. The same 
figure for the higher Reynolds number of $R e_{\mathrm{D}} \approx 17 \times 10^{3}$ is shown in Figure A2—due to high similarity, only the lower Reynolds number are discussed.

In all configurations shown, a distinct flow pattern develops showing the previously described pattern of separation lines between the repeating impingement-effusion cells. At the smallest cavity height $H=3 D$, the magnitude of downflow (e.g., negative velocity) is comparably small, the expected flow pattern is not developed completely, and balance fluxes exist throughout the flow cell. With increasing cavity height, the vortex system stabilizes and increases in downflow intensity. The separation lines appear more clearly. A stable vortex system establishes inside the cavity, clearly defining repeating flow cells with the optimal pattern to maximize the wetted area and optimize effusion hole inflow. For both alignments, the jet diameter increases with cavity height—-the absolute jet distance increases and thus, the jet will dissipate. This increases the area of jet impingement heat transfer on the effusion plate and reduces the local maximum values, which is also discussed in Figure 5.

Comparing the cases with and without misalignment, a breakup of the separation lines is visible. Due to the less ordered flow in the cavity and the different flow path lengths to the closest four effusion holes, the large cavity vortex is less stable. A stronger interaction between the adjacent flow cells is expected. Higher streamline curvature at the effusion hole inflow and stronger effusion hole inflow separation are the main results, also visible by increased unordered vector patterns in the effusion hole vicinity. Both lead to a decreased effusion hole flow and an increase in local heat transfer coefficients, especially at the effusion hole inflow edges. This effect is most pronounced for the smallest cavity $H=3 D$.

To further analyze the interaction between the impinging jet and the effusion hole inflow, a cutting plane is placed through the geometric centers of the two holes. Thus, for $M A L O 00$, this leads to a plane at an angle of $\approx 45^{\circ}$ to the $y$-normal plane, while for the misaligned cases, this resembles a $y$-normal plane. The resulting planes are shown in Figure 3 in green. For the rotated planes, the lateral coordinate, in-plane and out-of-plane velocities are recomputed via projection. The planar data are depicted in Figure 7 for the low Reynolds number $R e_{\mathrm{D}} \approx 10 \times 10^{3}$. The high Reynolds number data are shown in Figure A3 and again are not discussed in detail, due to high similarity. The plot shows the in-plane velocity using streamlines and the out-of-plane velocity using contours. All velocities are normalized, using the maximum velocity magnitude for a given plane.

For all aligned cases, left column, a clear flow vertical stratification to the two effusion holes is visible. No major areas of downflow are present; the majority of mass is transported down in the area in between the effusion holes and forms a stable vortex system. Mass is transported in and out of the plane at the bottom and top, respectively, as is also visible in Figure 6. Small recirculation areas are present for the smallest cavity height $H=3 D$ above $H^{*} \gtrsim 0.6$ on either side, showing the confined flow patterns with a less stable vortex system. Small inflow vortices are present for the configuration with the highest cavity $H=7 D$. Additionally, a second vortex system forms for $H=5 D$ and $H=7 D$ at the bottom, induced by jet drag. For the misaligned cases MALO05, shown in the right column, the flow patterns are different for each cavity height (especially for $x / D<0$, where the closest effusion inflow hole is located). For the smallest cavity $H=3 D$, the major flow direction upward is still maintained; however, a vortex system starts to develop at the left border. With increasing cavity height to $H=7 D$, this small pattern eventually forms a larger recirculation area with major downflow below the effusion inflow hole in the area of $x / D \approx-4$. The right region of the displayed figures $(x / D>0)$ show a more similar flow behavior. A vortex system forms that occupies a large region of the cavity at $x / D \approx 4$, where the next effusion inflow hole is located at $x / D=6.67$. The vortex system's height relative to the cavity height decreases with increasing cavity height. The previously described effects, combined, lead to a more complex and less systematic cavity flow, which in turn can influence the flow in the effusion holes, the separation behavior in the effusion hole diffusor and subsequently, the total cooling effectiveness on the hot gas side. 


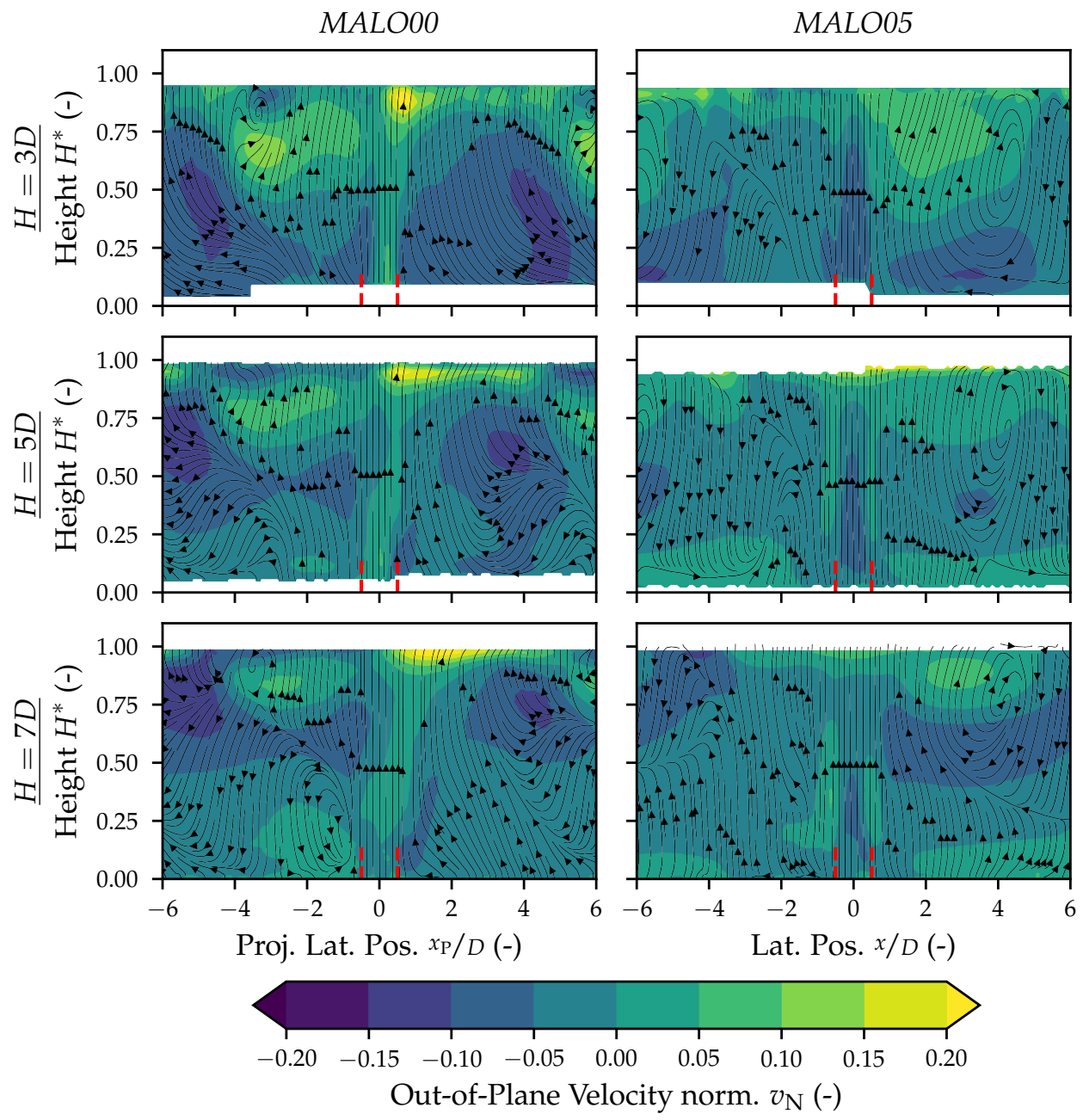

Figure 7. Cavity flow, low Reynolds number $R e_{\mathrm{D}} \approx 10 \times 10^{3}$. Planar cut data for the plane intersecting the impinging jet and the closest effusion hole, e.g., $\approx 45^{\circ}$ angle for MALO00 and $y$-normal for MALO05. Streamlines show in plane velocity, contours out of plane velocity. All velocities normalized with in-plane velocity magnitude. Impingement hole location shown in red.

\subsection{Transient Analysis}

The transient data are difficult to interpret, due to a large spread in time scales and noise induced by flow turbulence and measurement uncertainties. Thus, statistics and frequency domain analyses are employed to show the basic effects of instationary behavior. The jet flow statistic moments are summarized in Table 3 . For each configuration, the jet velocity magnitude is extracted at $H^{*} \uparrow=0.8$ and $H^{*} \downarrow=0.3$ at a line across the geometrical jet center with a width of $1.2 D$, and averaged. The velocity magnitude is normalized with the time-averaged maximum velocity magnitude at each plane. Eventually, the statistics are calculated.

The mean values are in good agreement with the previously shown jet profiles (due to the lateral average employed the jet profile effects are obscured). For the aligned cases $M A L O 00$ at $H=3 D$ and $H=5 D$, the integral momentum loss is comparatively small, and the jet can presumably expand freely. At the highest cavity $H=7 D$, the momentum loss is more substantial due to the extended jet travel distance and, additionally, the expanded jet profile is wider than the employed averaging area. However, these low velocity boundary regions will have no major effect on impingement heat transfer, justifying the area of data extraction. For the misaligned cases MALO05, the jet momentum loss is reduced (except $H=5 D, M A L O 00$; see also Figure 5) presumably due to the complex flow field in the cavity interacting with the jet flow profile. 
Table 3. Jet statistics at two different height locations for jet velocity magnitude, normalized with time-averaged maximum velocity magnitude at each configuration. Color shows magnitude relative to data in each major column (dark is low).

\begin{tabular}{|c|c|c|c|c|c|c|c|c|}
\hline \multirow{2}{*}{ Configuration } & \multicolumn{2}{|c|}{ Mean } & \multicolumn{2}{|c|}{ Std. Dev. $\left(\times 10^{2}\right)$} & \multicolumn{2}{|c|}{ Skew $\left(\times 10^{2}\right)$} & \multicolumn{2}{|c|}{ Kurtosis Exc. $\left(\times 10^{2}\right)$} \\
\hline & $H^{*} \uparrow$ & $H^{*} \downarrow$ & $H^{*} \uparrow$ & $H^{*} \downarrow$ & $H^{*} \uparrow$ & $H^{*} \downarrow$ & $H^{*} \uparrow$ & $H^{*} \downarrow$ \\
\hline 3D-MALO00 $R e_{\mathrm{D}} \downarrow$ & 0.46 & 0.59 & 8.5 & 4.6 & 2.9 & -14.1 & -36.9 & 28.0 \\
\hline 3D-MALO00 $\operatorname{Re}_{\mathrm{D}} \uparrow$ & 0.48 & 0.60 & 9.0 & 3.8 & -11.8 & -19.1 & -33.7 & 71.1 \\
\hline 3D-MALO05 $R e_{\mathrm{D}} \downarrow$ & 0.73 & 0.65 & 9.6 & 9.2 & -0.2 & 2.1 & -20.6 & -13.3 \\
\hline 3D-MALO05 $\operatorname{Re}_{\mathrm{D}} \uparrow$ & 0.69 & 0.64 & 8.7 & 8.5 & 2.3 & -32.7 & -26.6 & 0.9 \\
\hline 5D-MALO00 $R e_{\mathrm{D}} \downarrow$ & 0.65 & 0.72 & 8.3 & 8.6 & 10.9 & 32.4 & -11.4 & -0.9 \\
\hline $5 \mathrm{D}-M A L O 00 R e_{\mathrm{D}} \uparrow$ & 0.74 & 0.63 & 9.1 1 & 9.1 1 & 3.5 & 9.2 & -9.6 & -6.4 \\
\hline 5D-MALO05 $R e_{\mathrm{D}} \downarrow$ & 0.38 & 0.58 & 6.3 & 8.3 & 26.5 & -34.9 & 6.0 & -23.0 \\
\hline $5 \mathrm{D}-M A L O 05 \operatorname{Re}_{\mathrm{D}} \uparrow$ & 0.47 & 0.63 & 7.9 & 9.6 & 22.9 & -52.1 & 5.7 & 9.3 \\
\hline 7D-MALO00 $R e_{\mathrm{D}} \downarrow$ & 0.29 & 0.51 & 7.0 & 10.2 & 38.9 & 9.3 & 7.8 & -30.3 \\
\hline $7 \mathrm{D}-M A L O 00 R e_{\mathrm{D}} \uparrow$ & 0.32 & 0.52 & 7.6 & 9.9 & 25.8 & -10.8 & -10.8 & -37.7 \\
\hline 7D-MALO05 $R e_{\mathrm{D}} \downarrow$ & 0.68 & 0.76 & 9.2 & 9.7 & 0.5 & 12.2 & -21.2 & -7.3 \\
\hline 7D-MALO05 $R e_{\mathrm{D}} \uparrow$ & 0.74 & 0.78 & 9.4 4 & 9.7 & 2.6 & 27.9 & -3.7 & 2.8 \\
\hline
\end{tabular}

Standard deviations increase with impingement of the Reynolds number, due to the higher jet momentum and increased jet-crossflow interaction. This effect is more pronounced at the upper analysis line-jets with high initial momentum show less degradation and more variation further downstream. These systematic changes do not show for the lower cavity height $H=3 D$, where complex cavity flow patterns with higher momentum interact more strongly with the jet flow pattern. No clear connection between standard deviation and specimen alignment can be deduced.

Skew and Kurtosis excess show a less systematic behavior. Even though skew is normalized with the standard deviation, it is in general very low, showing an evenly distributed velocity magnitude (as expected for a presumably periodically varying jet). Kurtosis excess is notably different at the lower extraction for $H=3 D$ and $H=7 D$ at MALO00. The high values at the smallest cavity height point to smaller jet movement through the line of interest (less variation in velocity distribution width) and are again a confirmation for the confined flow in the cavity, where at high cavity heights, the jet can move in space and thus, leads to a wider spread in velocities observed at a fixed position.

Power Spectra

Power spectra for jet velocity are computed for both extraction lines. Additionally, a line is place just below the effusion hole inflow with a lateral width of $0.6 \mathrm{D}$ at $H^{*}=0.8$.

For all three cases, the main jet velocity component $(w)$ is chosen for frequency analysis. Frequencies are normalized as Strouhal numbers:

$$
S t=\frac{f D}{v_{\text {jet,e }}}
$$

with the computed jet exit velocity $v_{\text {jet,e }}$; see Table 2 . The amplitudes are normalized with the maximum amplitude in the spectrum. The resulting data are shown in Figure 8 for the reference case $H=5 D, M A L O 00$. Since the jet center plane does not intersect the effusion hole inflow plane, two different planes are selected for the analysis of the different spectra. All spectra are subject to noise for several reasons: the flow is turbulent, no main external excitation exists, and due to the Nyquist frequency at $f_{\mathrm{N}}=500 \mathrm{~Hz}$, parts of the spectrum are subject to either the mirror of shift frequencies (especially higher frequency turbulence may carry substantial energy). Additionally, any non-true periodic parts of the input signals cannot be represented, due to infinite-length time domain representation of the FFT approach. The jet spectra are similar for both Reynolds numbers. At the lower location, few distinct peaks exist, allowing the detection of a jet fluctuation Strouhal number. With 
increasing jet travel downstream, more peaks occur, and the jet movement increases in complexity. Jet fluctuation Strouhal numbers are expected to be in the order of $S t_{\text {jet }} \approx 0.02$. The effusion inflow spectrum shows a stronger skew to lower Strouhal numbers. This skew increases with the increase in the jet Reynolds number.

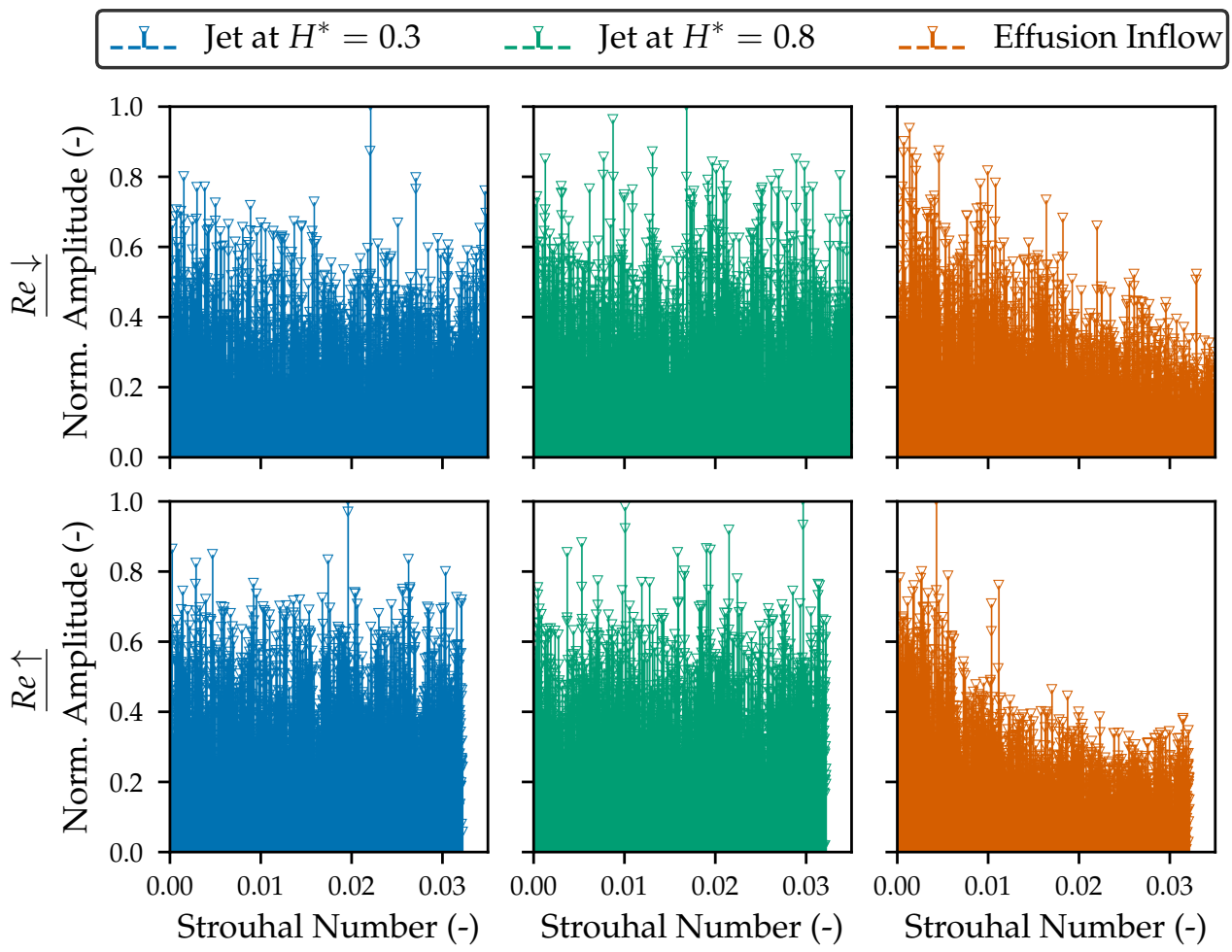

Figure 8. Frequency spectra displayed as Strouhal numbers at different locations for the two Reynolds numbers (rows). Configuration $H=5 D, M A L O 00$.

These spectra were analyzed for all configurations at the previously described positions. For each configuration and location, the four Strouhal numbers with the highest normalized (relative to the maximum for each configuration and each position) amplitudes are extracted and plotted in Figure 9, with the color showing the normalized amplitude.

Comparing all the configurations analyzed, the data are less systematic, due to the discussed shortcomings regarding noise and the (in-)correct detection of coherent structures. All configurations show the tendency of a less distinct dominant frequency when changing the evaluation section from $H^{*}=0.3$ to $H^{*}=0.8$ and subsequently, to the effusion inflow area, where the last step shows the major influence with the major frequencies collapsing at very low Strouhal numbers. Thus, jet fluctuation at higher frequencies are introduced to the jet by the coolant cross flow and the separation at the jet hole inlet (which is also the cause for the jet deviation). These higher frequency structures dissipate with time, and especially in the cavity flow, having the cavity act as a damper before the effusion hole inflow. This reduction in fluctuation can prove beneficial for a stable effusion cooling. For the aligned setups $M A L O 00$, a larger cavity height, especially $H=7 D$, increases damping. The difference between $H=3 D$ and $H=5 D$ is smaller. The misaligned configurations MALO05 show different behavior where the larger cavities allow wider spread of dominant Strouhal numbers, due to higher variation in secondary flow structures in the cavity. The smallest cavity height $H=3 D$ shows small differences between the aligned and misaligned case- - a sign that the hexagonal flow pattern cannot fully develop, even in the aligned cases and thus will not show significant differences to the misaligned cases. 


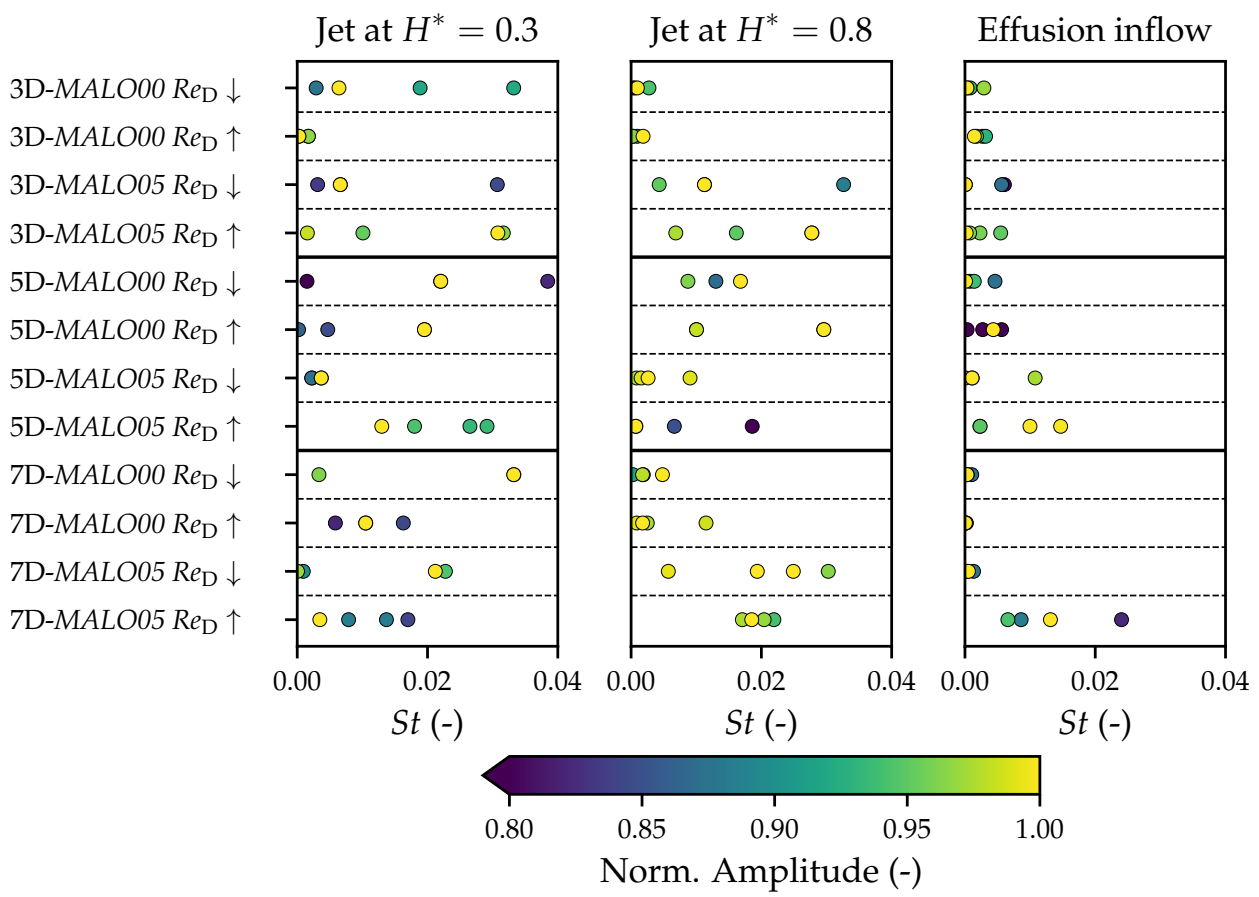

Figure 9. Frequency spectra at different locations for all configurations, using velocity magnitude as data signal. First four dominant frequencies shown. Color scale shows relative amplitude.

\section{Conclusions}

Extensive flow measurements were performed in the cavity of a double-wall combustor liner test rig. Data were presented for different jet Reynolds numbers $R e_{\mathrm{D}}$, different cavity heights and two different specimen alignments. In contrast to most of the previous research, coolant crossflow was introduced at realistic Reynolds numbers. In addition to time-averaged data, transient data were acquired and analyzed employing basic statistical methods and frequency analysis. Several conclusions can be drawn from the results.

The impinging jet profiles are in good agreement with previous research for similar setups. The main influence of cavity height shows in jet dissipation with jet flow distance. Thus, larger cavity heights will lead to a reduced jet momentum at the target wall; however, the induced heat transfer coefficient increase is spread over a larger area. As shown in the complimentary thermal data paper, this can lead to a more homogeneous thermal load distribution.

A jet deflection is visible in the opposite direction of the coolant crossflow, induced by a separation bubble at the impinging hole inflow. The resulting final jet deflection at the target wall again depends on the cavity height and jet Reynolds number. This deflection can change the location of the maximum impingement heat transfer.

Complex flow patterns are observed in the cavity. For the cavities with larger heights of $H=5 D$ and $H=7 D$ and aligned setups MALO00, a stable hexagonal vortex system with improved effusion inflow can be observed. This pattern is less pronounced with the smallest cavity height of $H=3 D$. The same pattern is disturbed by a misalignment of the two specimen, leading to a different, more chaotic cavity flow. This possible misalignment needs to be considered during design since thermal shift can lead to relative motion between the two walls of the combustor liner. 
Both analyzed operating points of $R e_{\mathrm{D}} \approx 10 \times 10^{3}$ and $R e_{\mathrm{D}} \approx 17 \times 10^{3}$ show similar results when comparing normalized data. Thus, the flow regime for both operating points is assumed to be similar, and the resulting effects on absolute values and, for example, heat transfer, can presumably be interpolated to operating points in between those bounds.

Initial transient data were analyzed, focusing on jet statistics and jet frequency spectra and effusion inflow spectra. Due to the complex time-dependent behavior, these basic methods only give limited insight into the transient flow mechanics. Jet velocity fluctuations increase with the jet Reynolds number and absolute jet traveling distance, and thus, the cavity height.

Basic frequency analysis showed that higher fluctuation frequencies are introduced into the cavity via the jet, their origin presumably being the separation bubble at the hole inflow and a general jet flow instability. The structures connected to those frequency dissipate with jet travel and with residence time in the cavity. At the effusion hole inflow (the cavity outflow), the spectra are shifted to lower frequencies, which can prove beneficial for stable effusion cooling.

For all analyzed values, the configuration with the lowest cavity height $H=3 D$ showed the highest spread in results, presumably due to the increased flow complexity and instability in the confined cavity.

It has to be noted that the analysis of power spectra was also performed on data without heat addition. The jet spectra are assumed to show little variation with the addition of heat transfer since they are mainly influenced by the cooling air flow. The cavity and effusion spectra are assumed to be more susceptible to change in the case of heat addition. Future analyses are planned.

To better identify dominant frequencies, and more importantly, coherent structures, advanced methods need to be applied. Dominant frequencies are obscured due to damping and non-periodic frequency changes. The application of either short time Fourier transform or wavelet transform may mitigate this issue. Additionally, using proper orthogonal decomposition or dynamic mode decomposition can yield more insight into the complex transient flow coherent structures. After the identification of coherent structures, frequency analysis can be performed more specifically, eliminating most of the noise effects.

Author Contributions: T.J., M.E. and H.-J.B. have contributed equally to this version of the manuscript. The work was prepared in close collaboration between the authors, and there is no strict separation between text passages. All authors have read and agreed to the published version of the manuscript. K.S. and M.H. supported the review and editing of the manuscript.

Funding: This project was supported by the Federal Ministry for Economic Affairs and Energy, Germany in the framework of LuFo—the Federal Aeronautical Research Programme. We acknowledge support by the KIT-Publication Fund of the Karlsruhe Institute of Technology.

Institutional Review Board Statement: Not applicable.

Informed Consent Statement: Not applicable.

Data Availability Statement: The data presented in this study are available on request from the corresponding author. The data are not publicly available, due to the large amounts of data. In addition to the aerodynamic results presented in this article, thermal data (infrared thermography) are also available for the same test setup.

Conflicts of Interest: The authors declare no conflict of interest. 


\section{Abbreviations}

The following abbreviations and symbols are used in this manuscript:

\section{Symbols}

$\Delta$

$\alpha$

$\beta$

$\lambda$

$v$

D

$D R$

E

$f$

$H$

$H^{*}$

I

M

$N$

$P$

$S$

SF

$T$

$\mathrm{Tu}$

$t$

$v$

$\{u, v, w\}$

W

Abbreviations

ITS

MALO

PEEK

PIV

PTFE

$R e$

RQL

St

Scripts

$\infty$

c

d

des

e

f

eff

$\mathrm{h}$

imp

int

ref

tot

L

N

$\mathrm{O}$

\section{Difference}

Hole inclination

Laidback and fan-shape angle

Wavelength

Viscosity

Reference Diameter, Diameter

Density Ratio

Effusion hole diameter

Frequency

Height

Normalized Height

Momentum Ratio

Blowing Ratio $M$

Number of Holes

Pitch, Power

Shift

Scaling factor

Temperature

Turbulence level

Thickness

Velocity

Velocity components

Width

Institute of Thermal Turbomachinery

Longitudinal Misalignment

Polyetheretherketone

Particle Image Velocimetry

Teflon ${ }^{\mathrm{TM}}$

Reynolds number

Rich-Quench-Lean

Strouhal number

free stream

cold

Data rate

design

effective

Frequency

Effusion

hot

Impingement

internal

reference

total

laidback (angle)

Normalized, Nyquist

opening (angle) 


\section{Appendix A. Additional Data}



Figure A1. Jet profiles for all configurations tested. Profiles show normalized velocity at three different height (colors) for both alignments (line style) at both Reynolds numbers (rows) and different cavity heights (columns). Velocities taken at $x$-normal planes. Misaligned cases shifted longitudinally for visualization. 

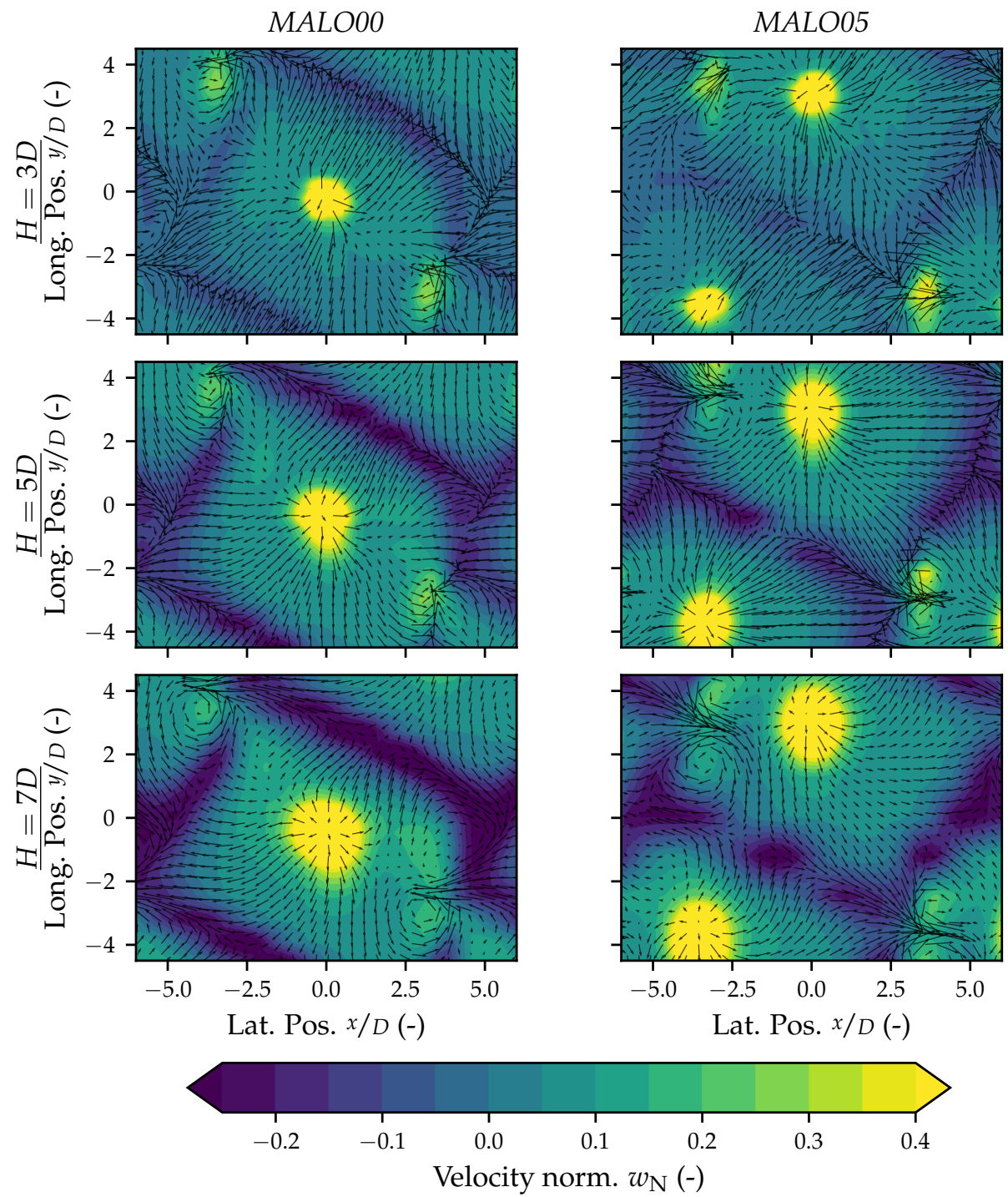

Figure A2. Cavity flow, low Reynolds number $R e_{\mathrm{D}} \approx 17 \times 10^{3}$. w-velocity normalized shown at $z$-normal plane with $H^{*}=80 \%$. Vectors show planar velocity components, unit length $v e c_{\mathrm{ul}}=3$. Rows show cavity height, columns show alignment. 


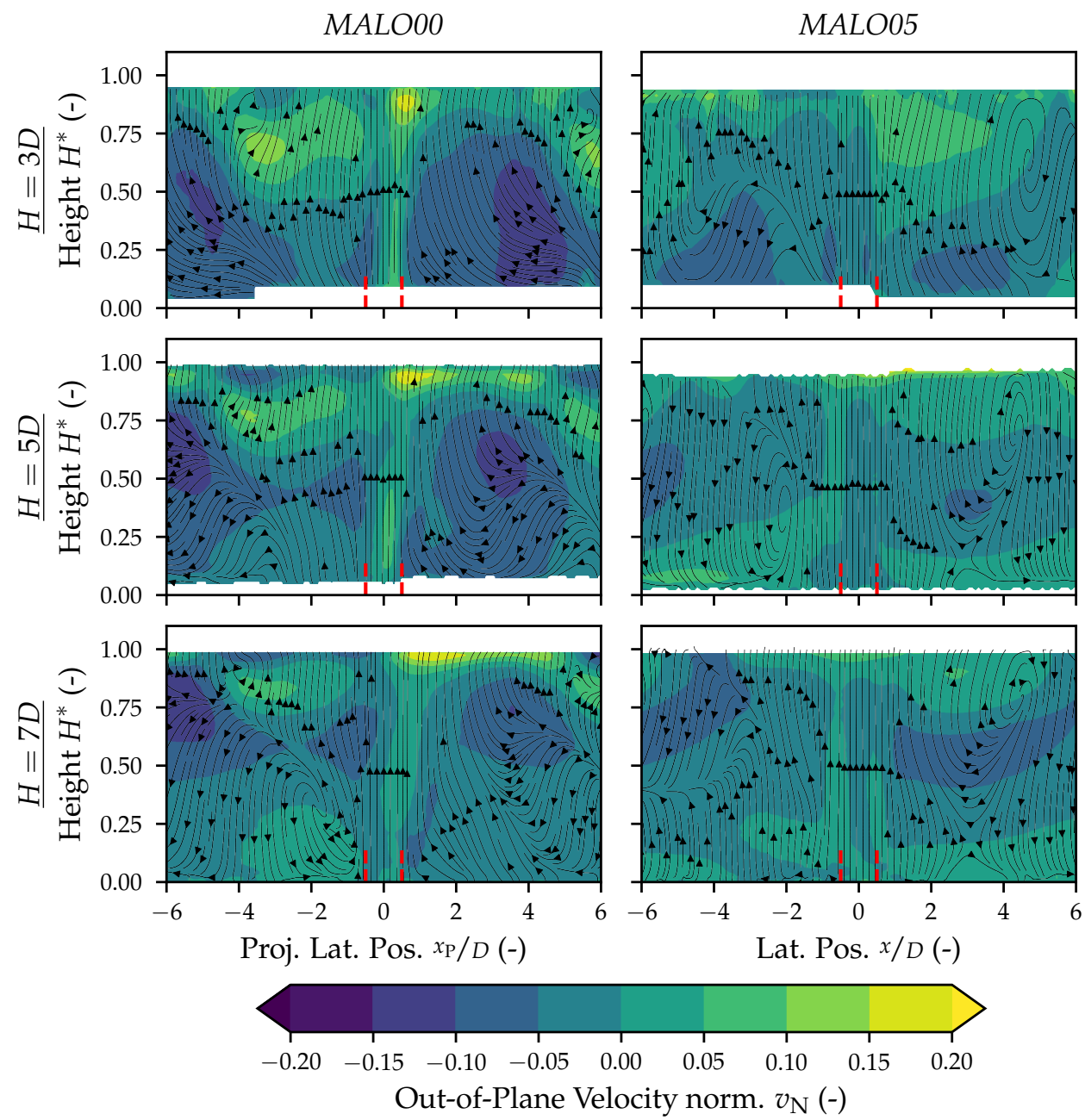

Figure A3. Cavity flow, high Reynolds number $R e_{\mathrm{D}} \approx 17 \times 10^{3}$. Planar cut data for the plane intersecting the impinging jet and the closest effusion hole, e.g., $\approx 45^{\circ}$ angle for MALO00 and $y$-normal for MALO05. Streamlines shown in plane velocity, contours out of plane velocity. All velocities normalized with in-plane velocity magnitude. Impingement hole location shown in red.

\section{Appendix B. Literature Review}

Table A1. Overview of references with similar research. The marked cells indicate whether the individual reference attend to different flow parameters, cooling similarities, the double-wall combustor geometry and thermal or aerodynamic data availability. For this overview, the details of available data are not considered, e.g., most of the aerodynamic data comprise the discharge coefficients and pressure losses with no detailed flow field as presented in this study.

\begin{tabular}{|c|c|c|c|c|c|c|c|c|}
\hline \multirow{2}{*}{ Reference } & \multicolumn{3}{|c|}{ Flow } & \multicolumn{2}{|c|}{ Cooling } & \multirow[t]{2}{*}{ Geometry } & \multicolumn{2}{|c|}{ Data } \\
\hline & $R e_{\mathrm{c}}$ & $R e_{\mathrm{h}}$ & $C C^{\dagger}$ & $D R^{+}$ & $I^{\dagger}$ & & Thermal & Aero \\
\hline This study and [2] & $\bullet$ & $\bullet$ & $\bullet$ & $\bullet$ & $\bullet$ & $\bullet$ & $\bullet$ & $\bullet$ \\
\hline Albert et al. [24] & & $\bullet$ & & $\bullet$ & $\bullet$ & & $\bullet$ & \\
\hline Al Dabagh et al. [7] & - & & - & - & - & & - & - \\
\hline Alenezi et al. [19] & - & & & & & & - & - \\
\hline Anderson et al. [25] & - & & & - & - & & - & \\
\hline Andreini et al. [26] & $\bullet$ & $\bullet$ & & $\bullet$ & $\bullet$ & $\bullet$ & $\bullet$ & \\
\hline Andrews et al. [27] & - & & - & - & - & & - & \\
\hline Andrews et al. [3] & $\bullet$ & & $\bullet$ & $\bullet$ & $\bullet$ & & $\bullet$ & \\
\hline
\end{tabular}


Table A1. Cont.

\begin{tabular}{|c|c|c|c|c|c|c|c|c|}
\hline \multirow{2}{*}{ Reference } & \multicolumn{3}{|c|}{ Flow } & \multicolumn{2}{|c|}{ Cooling } & \multirow[t]{2}{*}{ Geometry } & \multicolumn{2}{|c|}{ Data } \\
\hline & $R e_{\mathrm{c}}$ & $R e_{\mathrm{h}}$ & $C C^{+}$ & $D R^{\dagger}$ & $I^{\dagger}$ & & Thermal & Aero \\
\hline Bailey et al. [28] & $\bullet$ & • & - & & $\bullet$ & - & $\bullet$ & $\bullet$ \\
\hline Bunker [29] & $\bullet$ & & & $\bullet$ & $\bullet$ & & $\bullet$ & $\bullet$ \\
\hline Chen et al. [9] & $\bullet$ & & $\bullet$ & & & $\bullet$ & $\bullet$ & $\bullet$ \\
\hline Click et al. [11] & $\bullet$ & $\bullet$ & $\bullet$ & & $\bullet$ & $\bullet$ & $\bullet$ & $\bullet$ \\
\hline Cocchi et al. [8] & $\bullet$ & & $\bullet$ & & & $\bullet$ & $\bullet$ & $\bullet$ \\
\hline El-Jummah et al. [5] & • & & - & • & • & & $\bullet$ & $\bullet$ \\
\hline Florschuetz et al. [30] & $\bullet$ & & $\bullet$ & & & & - & • \\
\hline Huelsmann et al. [31] & - & & & & & & $\bullet$ & \\
\hline Jackowski et al. [20] & - & $\bullet$ & & • & - & • & $\bullet$ & - \\
\hline Kaskade et al. [32] & $\bullet$ & $\bullet$ & $\bullet$ & & $\bullet$ & $\bullet$ & $\bullet$ & $\bullet$ \\
\hline Lazik et al. [33] & & & & & & $\bullet$ & $\bullet$ & $\bullet$ \\
\hline Ligrani et al. [34] & $\bullet$ & $\bullet$ & & & $\bullet$ & $\bullet$ & $\bullet$ & \\
\hline Ligrani et al. [10] & $\bullet$ & $\bullet$ & $\bullet$ & & $\bullet$ & $\bullet$ & $\bullet$ & \\
\hline Lin et al. [35] & & & $\bullet$ & & & & $\bullet$ & $\bullet$ \\
\hline Martiny et al. [4] & $\bullet$ & $\bullet$ & & $\bullet$ & $\bullet$ & $\bullet$ & $\bullet$ & \\
\hline Murray et al. [36] & & & & & & $\bullet$ & $\bullet$ & \\
\hline Murray et al. [13] & & $\bullet$ & & & & $\bullet$ & $\bullet$ & $\bullet$ \\
\hline Murray et al. [14] & $\bullet$ & $\bullet$ & $\bullet$ & $\bullet$ & & $\bullet$ & $\bullet$ & \\
\hline Nathan et al. [37] & $\bullet$ & • & & $\bullet$ & $\bullet$ & & $\bullet$ & \\
\hline Ngetich et al. [38] & & $\bullet$ & & $\bullet$ & $\bullet$ & & $\bullet$ & \\
\hline Ngetich et al. [39] & & $\bullet$ & & $\bullet$ & & & $\bullet$ & $\bullet$ \\
\hline O’Donovan et al. [17] & $\bullet$ & & & & & & $\bullet$ & $\bullet$ \\
\hline O’Donovan et al. [18] & $\bullet$ & & & & & & • & $\bullet$ \\
\hline Oguntade et al. [6] & & & $\bullet$ & $\bullet$ & & $\bullet$ & $\bullet$ & \\
\hline Ren et al. [40] & $\bullet$ & $\bullet$ & $\bullet$ & & $\bullet$ & $\bullet$ & $\bullet$ & \\
\hline Rogers et al. [41] & $\bullet$ & & $\bullet$ & & & $\bullet$ & $\bullet$ & \\
\hline Schroeder and Thole [22] & & $\bullet$ & & • & $\bullet$ & & - & \\
\hline Shrager et al. [15] & & & & & & $\bullet$ & $\bullet$ & \\
\hline Shrager et al. [16] & & & & & & $\bullet$ & & $\bullet$ \\
\hline Sweeney and Rhodes [42] & & $\bullet$ & & $\bullet$ & & $\bullet$ & $\bullet$ & \\
\hline Vanga et al. [12] & $\bullet$ & $\bullet$ & $\bullet$ & & $\bullet$ & $\bullet$ & $\bullet$ & \\
\hline Vanga et al. [43] & • & • & • & & • & • & • & \\
\hline
\end{tabular}




\section{Appendix C. Reference Values}

Table A2. Reference values for normalized plot quantities.

\begin{tabular}{|c|c|c|c|c|c|c|c|c|c|c|c|c|}
\hline \multirow{3}{*}{ Figure } & \multicolumn{6}{|c|}{$\operatorname{Re}_{\mathrm{D}} \approx 10 e 3$} & \multicolumn{6}{|c|}{$R e_{\mathrm{D}} \approx 17 e 3$} \\
\hline & \multicolumn{3}{|c|}{ MALO00 } & \multicolumn{3}{|c|}{ MALO05 } & \multicolumn{3}{|c|}{ MALOOO } & \multicolumn{3}{|c|}{ MALO05 } \\
\hline & $3 D$ & $5 D$ & $7 D$ & $3 D$ & $5 D$ & $7 D$ & $3 D$ & $5 D$ & $7 D$ & $3 D$ & $5 D$ & $7 D$ \\
\hline Figure $5(\mathrm{~m} / \mathrm{s})$ & 47.64 & 46.50 & 48.17 & 48.10 & 46.97 & 44.13 & 71.54 & 66.90 & 74.09 & 72.34 & 62.81 & 73.31 \\
\hline Figure A1 $(\mathrm{m} / \mathrm{s})$ & 46.38 & 47.03 & 50.42 & 48.10 & 46.25 & 42.30 & 71.02 & 65.27 & 74.07 & 72.31 & 63.62 & 72.57 \\
\hline Figure $6(\mathrm{~m} / \mathrm{s})$ & 24.42 & 22.29 & 21.44 & 21.09 & 23.06 & 22.00 & & & & & & \\
\hline Figure A2 $(\mathrm{m} / \mathrm{s})$ & & & & & & & 40.07 & 37.75 & 34.93 & 37.08 & 37.31 & 36.38 \\
\hline Figure $7(\mathrm{~m} / \mathrm{s})$ & 46.08 & 47.15 & 49.59 & 48.10 & 46.97 & 44.13 & & & & & & \\
\hline Figure A3 $(\mathrm{m} / \mathrm{s})$ & & & & & & & 70.10 & 63.05 & 73.45 & 72.34 & 62.81 & 73.31 \\
\hline
\end{tabular}

\section{References}

1. Lefebvre, A.H.; Ballal, D.R. Gas Turbine Combustion: Alternative Fuels and Emissions, 3rd ed.; Taylor \& Francis: Boca Raton, FL, USA, 2010

2. Jackowski, T.; Elfner, M.; Bauer, H.J. Experimental Study of Impingement Effusion-Cooled Double-Wall Combustor Liners: Thermal Analysis. Energies 2021, 14, 4843. [CrossRef]

3. Andrews, G.E.; Asere, A.A.; Hussain, C.I.; Mkpadi, M.C.; Nazari, A. Impingement/Effusion Cooling: Overall Wall Heat Transfer In Proceedings of the ASME 1988 International Gas Turbine and Aeroengine Congress, Amsterdam, The Netherlands, 6 June 1988; p. V004T09A036. [CrossRef]

4. Martiny, M.; Schulz, A.; Wittig, S. Effusion Cooled Combustor Liners of Gas Turbines-An Assessment of the Contributions of Convective, Impingement, and Film Cooling. In Proceedings of the Symposium on Energy Engineering in the 21st Century (SEE2000), Hong Kong, China, 9-13 January 2000; pp. 221-228.

5. El-Jummah, A.; Nazari, A.; Andrews, G.E.; Staggs, J.E. Impingement/Effusion Cooling Wall Heat Transfer: Reduced Number of Impingement Jet Holes Relative to the Effusion Holes. In Proceedings of the ASME Turbo Expo, 2017, Charlotte, NC, USA, 26-30 June 2017; p. GT2017-63494. [CrossRef]

6. $\quad$ Oguntade, H.I.; Andrews, G.E.; Burns, A.D.; Ingham, D.B.; Pourkashanian, M. Impingement/Effusion Cooling with Low Coolant Mass Flow. In Proceedings of the ASME Turbo Expo, 2017, Charlotte, NC, USA, 26-30 June 2017; p. GT2017-63484. [CrossRef]

7. Al Dabagh, A.M.; Andrews, G.E.; Abdul Husain, R.A.A.; Husain, C.I.; Nazari, A.; Wu, J. Impingement/Effusion Cooling: The Influence of the Number of Impingement Holes and Pressure Loss on the Heat Transfer Coefficient. J. Turbomach. 1990, 112, 467. [CrossRef]

8. Cocchi, L.; Picchi, A.; Mazzei, L.; Andreini, A.; Belloci, L. Effect of Holes Arrangement on Heat Transfer in Impingement/Effusion Cooling Double Wall Schemes. In Proceedings of the 1st GPPS, Zurich, Switzerland, 16-18 January 2017; p. GPPF-2017-77.

9. Chen, G.; Liu, Y.; Rao, Y.; He, J.; Qu, Y. Numerical Investigation on Conjugate Heat Transfer of Impingement/Effusion Double-Wall Cooling with Different Crossflow Schemes. Appl. Therm. Eng. 2019, 155, 515-524. [CrossRef]

10. Click, A.; Ritchie, D.; Ligrani, P.M.; Liberatore, F.; Patel, R.; Ho, Y.H. Double Wall Cooling of an Effusion Plate with Cross Flow and Impingement Jet Combination Internal Cooling: Comparisons of Main Flow Contraction Ratio Effects. Int. J. Heat Mass Transf. 2020, 150, 119196. [CrossRef]

11. Click, A.; Ligrani, P.; Ritchie, D.; Liberatore, F.; Patel, R.; Ho, Y.H. Effects of Coolant Supply Arrangement on Double Wall Cooling: Hot-side Effusion Performance and Cold-Side Nusselt Numbers at Different Initial Blowing Ratios. Int. J. Heat Mass Transf. 2020, 156, 119808. [CrossRef]

12. Vanga, S.R.; Ritchie, D.; Click, A.; Ren, Z.; Ligrani, P.; Liberatore, F.; Patel, R.; Srinivasan, R.; Ho, Y.H. Double Wall Cooling of a Full-Coverage Effusion Plate with Main Flow Pressure Gradient, Including Internal Impingement Array Cooling. J. Turbomach. 2019, 141, 041002. [CrossRef]

13. Murray, A.V.; Ireland, P.T.; Romero, E. Development of a Steady-State Experimental Facility for the Analysis of Double-Wall Effusion Cooling Geometries. J. Turbomach. 2019, 141, 041008. [CrossRef]

14. Murray, A.V.; Ireland, P.T.; Romero, E. Experimental and Computational Methods for the Evaluation of Double-Wall, Effusion Cooling Systems. J. Turbomach. 2020, 142, 111003. [CrossRef]

15. Shrager, A.C.; Thole, K.A.; Mongillo, D. Effects of Effusion Cooling Pattern Near the Dilution Hole for a Double-Walled Combustor Liner-Part 1: Overall Effectiveness Measurements. J. Eng. Gas Turbines Power 2019, 141, 10. [CrossRef]

16. Shrager, A.C.; Thole, K.A.; Mongillo, D. Effects of Effusion Cooling Pattern Near the Dilution Hole for a Double-Walled Combustor Liner-Part II: Flowfield Measurements. J. Eng. Gas Turbines Power 2019, 141, 10. [CrossRef]

17. O’Donovan, T.S.; Murray, D.B. Jet Impingement Heat Transfer-Part I: Mean and Root-Mean-Square Heat Transfer and Velocity Distributions. Int. J. Heat Mass Transf. 2007, 50, 3291-3301. [CrossRef] 
18. O'Donovan, T.S.; Murray, D.B. Jet Impingement Heat Transfer-Part II: A Temporal Investigation of Heat Transfer and Local Fluid Velocities. Int. J. Heat Mass Transf. 2007, 50, 3302-3314. [CrossRef]

19. Alenezi, A.H.; Almutairi, A.; Alhajeri, H.M.; Addali, A.; Gamil, A.A. Flow Structure and Heat Transfer of Jet Impingement on a Rib-Roughened Flat Plate. Energies 2018, 11, 1550. [CrossRef]

20. Jackowski, T.; Elfner, M.; Bauer, H.J. Numerical Investigation of Conjugate Heat Transfer in an Effusion and Impingement Cooled Combustor Wall. In Proceedings of the ASME Turbo Expo, 2020, London, UK (Virtual Event), 22-26 June 2020; p. GT2020-15505.

21. Roach, P. The Generation of Nearly Isotropic Turbulence by Means of Grids. Int. J. Heat Fluid Flow 1987, 8, 82-92. [CrossRef]

22. Schroeder, R.P.; Thole, K.A. Adiabatic Effectiveness Measurements for a Baseline Shaped Film Cooling Hole. In Proceedings of the Turbo Expo: Power for Land, Sea, and Air, 2014, Duesseldorf, Germany, 16 June 2014. [CrossRef]

23. Wieneke, B. PIV uncertainty quantification from correlation statistics. Meas. Sci. Technol. 2015, 26, 074002. [CrossRef]

24. Albert, J.E.; Bogard, D.G.; Cunha, F. Adiabatic and Overall Effectiveness for a Film Cooled Blade. In Proceedings of the Turbo Expo: Power for Land, Sea, and Air, 2004, Vienna, Austria, 14-17 June 2004. [CrossRef]

25. Anderson, J.B.; Wilkes, E.K.; McClintic, J.W.; Bogard, D.G. Effects of Freestream Mach Number, Reynolds Number, and Boundary Layer Thickness on Film Cooling Effectiveness of Shaped Holes. In Proceedings of the Turbo Expo: Power for Land, Sea, and Air, 2016, Seoul, South Korea, 13-17 June 2016; Volume 5C. [CrossRef]

26. Andreini, A.; Caciolli, G.; Facchini, B.; Tarchi, L.; Coutandin, D.; Taddei, S.; Peschiulli, A. Combustor Liner Cooled by a Combined Slot/Effusion System. In Proceedings of the ASME Turbo Expo, 2012, Copenhagen, Denmark, 11-15 June 2012; p. GT2012-68263. [CrossRef]

27. Andrews, G.; Asere, A.; Gupta, M.; Mkpadi, M. Full Coverage Discrete Hole Film Cooling: The Influence of Hole Size. Int. J. Turbo Jet Engines 1985, 2. [CrossRef]

28. Bailey, J.C.; Intile, J.; Fric, T.F.; Tolpadi, A.K.; Nirmalan, N.V.; Bunker, R.S. Experimental and Numerical Study of Heat Transfer in a Gas Turbine Combustor Liner. J. Eng. Gas Turbines Power 2003, 125, 994. [CrossRef]

29. Bunker, R.S. A Review of Shaped Hole Turbine Film-Cooling Technology. J. Heat Transf. 2005, 127, 441-453. [CrossRef]

30. Florschuetz, L.W.; Truman, C.R.; Metzger, D.E. Streamwise Flow and Heat Transfer Distributions for Jet Array Impingement with Crossflow. In Proceedings of the Turbo Expo: Power for Land, Sea, and Air, 1981, Houston, TX, USA, 9-12 March 1981. [CrossRef]

31. Huelsmann, N.C.; Thole, K.A. Effects of Jet Impingement on Convective Heat Transfer in Effusion Holes. J. Turbomach. 2021, 143, 10. [CrossRef]

32. Kakade, V.U.; Thorpe, S.J.; Gerendás, M. Effusion-Cooling Performance at Gas Turbine Combustor Representative Flow Conditions. In Proceedings of the Turbo Expo: Power for Land, Sea, and Air, 2012, Copenhagen, Denmark, 11-15 June 2012; Volume 4. [CrossRef]

33. Lazik, W.; Doerr, T.; Bake, S.; Bank, R.v.d.; Rackwitz, L. Development of Lean-Burn Low-NOx Combustion Technology at Rolls-Royce Deutschland. In Proceedings of the Turbo Expo: Power for Land, Sea, and Air, 2008, Berlin, Germany, 9-13 June 2008; Volume 3. [CrossRef]

34. Ligrani, P.; Ren, Z.; Liberatore, F.; Patel, R.; Srinivasan, R.; Ho, Y.H. Double Wall Cooling of a Full-Coverage Effusion Plate, Including Internal Impingement Array Cooling. J. Eng. Gas Turbines Power 2017, 140. [CrossRef]

35. Lin, Y.; Song, B.; Li, B.; Liu, G.; Wu, Z. Investigation of Film Cooling Effectiveness of Full-Coverage Inclined Multihole Walls With Different Hole Arrangements. In Proceedings of the Turbo Expo: Power for Land, Sea, and Air, 2003, Atlanta, GA, USA, 16-19 June 2003; Volume 5. [CrossRef]

36. Murray, A.V.; Ireland, P.T.; Rawlinson, A.J. An Integrated Conjugate Computational Approach for Evaluating the Aerothermal and Thermomechanical Performance of Double-Wall Effusion Cooled Systems. In Proceedings of the ASME Turbo Expo, 2017, Charlotte, NC, USA, 26-30 June 2017; p. GT2017-64711. [CrossRef]

37. Nathan, M.L.; Dyson, T.E.; Bogard, D.G.; Bradshaw, S.D. Adiabatic and Overall Effectiveness for the Showerhead Film Cooling of a Turbine Vane. J. Turbomach. 2013, 136, 031005. [CrossRef]

38. Ngetich, G.C.; Ireland, P.T.; Romero, E. Study of Film Cooling Effectiveness on a Double-Walled Effusion-Cooled Turbine Blade in a High-Speed Flow Using Pressure Sensitive Paint. In Proceedings of the ASME Turbo Expo, 2019, Phoenix, AZ, USA, 17-21 June 2019; p. GT2019-90545. [CrossRef]

39. Ngetich, G.C.; Murray, A.V.; Ireland, P.T.; Romero, E. A Three-Dimensional Conjugate Approach for Analysing a Double-Walled Effusion-Cooled Turbine Blade. J. Turbomach. 2019, 141, 011002. [CrossRef]

40. Ren, Z.; Vanga, S.R.; Rogers, N.; Ligrani, P.; Hollingsworth, K.; Liberatore, F.; Patel, R.; Srinivasan, R.; Ho, Y.H. Internal and External Cooling of a Full Coverage Effusion Cooling Plate: Effects of Double Wall Cooling Configuration and Conditions. In Proceedings of the ASME Turbo Expo, 2017, Charlotte, NC, USA, 26-30 June 2017; Volume 5A. [CrossRef]

41. Rogers, N.; Ren, Z.; Buzzard, W.; Sweeney, B.; Tinker, N.; Ligrani, P.M. Effects of Double Wall Cooling Configuration and Conditions on Performance of Full Coverage Effusion Cooling. In Proceedings of the ASME Turbo Expo, 2016, Seoul, South Korea, 13-17 June 2016; p. GT2016-56515. [CrossRef]

42. Sweeney, P.C.; Rhodes, J.F. An Infrared Technique for Evaluating Turbine Airfoil Cooling Designs. J. Turbomach. 1999, $122,170-177$. [CrossRef]

43. Vanga, S.R.; Ligrani, P.M.; Knox, J.; Liberatore, F.; Patel, R.; Ho, Y.H. Louver and effusion cooling heat transfer for a double wall effusion plate with impingement jet array coolant supply. Int. J. Heat Mass Transf. 2021, 168, 120861. [CrossRef] 